\title{
The Use of Computed Tomography of Pulmonary Angiogram in a District Hospital
}

\author{
Bomi Kim, ${ }^{1}$ Matthew Hills, ${ }^{1}$ and Lutz Beckert ${ }^{2}$ \\ ${ }^{1}$ Department of Medicine, Timaru Hospital, Private Bag 911, Queen Street, Timaru 7910, New Zealand \\ ${ }^{2}$ Department of Respiratory Medicine, Christchurch Hospital, Riccarton Avenue, P.O. Box 4710, Christchurch 8140, New Zealand
}

Correspondence should be addressed to Matthew Hills; mhills@timhosp.co.nz

Received 14 August 2013; Accepted 12 September 2013

Academic Editors: P. Schoenhagen and H. Yoshida

Copyright (C) 2013 Bomi Kim et al. This is an open access article distributed under the Creative Commons Attribution License, which permits unrestricted use, distribution, and reproduction in any medium, provided the original work is properly cited.

\begin{abstract}
Background. Computed tomography of pulmonary angiogram (CTPA) is a widely used investigation in patients with suspected pulmonary embolism (PE). It is not without adverse effects either through contrast injection or radiation exposure. International guidelines suggest that patients with a low/intermediate clinical probability and negative D-dimer do not require CTPA to exclude PE. Method. A retrospective audit of 100 consecutive CTPA scans was performed at a district general hospital from January to May 2012 to assess adherence to the current guidelines for diagnosis of acute PE and utilisation of CTPA. Result. Of the total 88 CTPA scans that were included in the study, $14 \%$ were positive for PE. At least $6 \%$, potentially up to $30 \%$ of all the scans, could have been avoided by adherence to current guidelines, thereby improving the positive yield to as high as $19 \%$. Clinical probability scores were documented in only $3 \%$. Orthopaedics requested $3 \%$ of all scans and the Assessment Treatment and Rehabilitation (ATR) unit had the highest number of CTPA requests per 100 departmental inpatient admissions. Conclusion. Adherence to the current guidelines can reduce the number of CTPA scans required and may reduce cost, contrast exposure, and radiation burden.
\end{abstract}

\section{Introduction}

Computed Tomography Pulmonary Angiography (CTPA) is the most commonly employed investigation in patients with suspected acute pulmonary embolism (PE). Most evidence is derived from the large University Hospitals in North America. Most scans are performed in smaller centres around the world. CTPA scanning is convenient as it is accessible after hours and it can often offer alternative diagnoses; however, its disadvantages includes cost, contrast use, and radiation exposure $[1,2]$. In a theoretical analysis, it was estimated that current typical testing practices for PE in the Emergency Department would prevent six deaths and 24 major nonfatal PE events, but also cause 36 deaths and 37 nonfatal major harms per 10,000 from contrast-induced renal failure, radiation-induced cancer, and major haemorrhage from anticoagulation [1].

Current guidelines based on studies in tertiary centres suggest using a clinical probability score (Wells criteria or Geneva score) and D-dimer testing prior to requesting CTPA. The pivotal aspects of these guidelines in CTPA-experienced institutions with quantitative D-dimer assay are $[2,3]$ as follows.

(1) Low/intermediate clinical probability and negative Ddimer excludes PE without CTPA.

(2) Low/intermediate clinical probability and positive Ddimer needs CTPA to confirm.

(3) High clinical probability needs CTPA to confirm without need for D-dimer.

We sought to audit adherence to the current guidelines and assess utilisation of CTPA in our small district General Hospital.

\section{Methods}

Timaru hospital is a 131-bed regional hospital serving a population of approximately 50,000 people in South Canterbury. It used a multidetector 4-slice CT scanner during the study period. Using InteleViewer software, 100 consecutive CTPA 
scans performed from January to May 2012 were selected for retrospective analysis.

Clinical probability scores (simplified Wells score and revised Geneva score) were calculated retrospectively by reviewing the patient notes. D-dimer values were collected from the hospital's computerised results system. Timaru Hospital uses quantitative immunoturbidimetric D-dimer assay (STA-Liatest D-Di), and its negative predictive value is reported to be in $98-100 \%$ range with the cut-off value of $500 \mathrm{mcg} / \mathrm{L}$ FEU (Fibrinogen Equivalent Unit) [4]. The clinical probability scores and D-dimer values were then analysed against CTPA results.

This audit does not require formal ethics approval according to the New Zealand Health and Disability Commissioner guidelines.

\section{Results}

After excluding 11 medical outpatient scans and one scan of a patient whose data were not available at the time of analysis, a total of 88 CTPA scans were reviewed.

The age of patients ranged from 21 to 94 years, with a mean age of 67 years. Approximately 53\% of the scans were performed in women. Of all 88 scans, 43 (49\%) were requested by the Emergency Department, 27 (31\%) by the General Medicine, 9 (10\%) by the General Surgery, and the rest by Assessment Treatment and Rehabilitation (ATR), Orthopaedics, and Obstetrics and Gynaecology (O\&G) (Figure 1). The Assessment Treatment and Rehabilitation unit provides health care services for the elderly and aims to assist with their recovery from illness and reintegrate them into the community.

Relative to the total number of admissions in each department, ATR had the highest rate of CTPA requests4.24 scans per 100 admissions, which is five times the average rate (Table 1). Of all scans, only three (3\%) cases had the clinical probability scores documented in the patient notes. A total $25 \%$ of the scans were performed after hours.

The reported findings for the 88 CTPA scans were PE in 12 (14\%) scans, alternative diagnoses in 44 (50\%) scans, and no abnormality identified in 32 (36\%) scans (Figure 2). The audit also notes that $2 \%$ of the scans were nondiagnostic and $22 \%$ were of substandard quality.

Stratification of the CTPA scans by combining the retrospectively calculated simplified Wells criteria with the D-dimer result using the conventional cut-off value of $500 \mathrm{mcg} / \mathrm{L}$ FEU (Table 2) showed that 18 (20\%) of them had low probability scores without D-dimer testing; 5 (6\%) had low scores and negative D-dimer. Similar results were noted when the Revised Geneva scoring system was used instead 23 (26\%) scored low/intermediate; however, D-dimer testing was not performed; 5 (6\%) had low/intermediate scores and had negative D-dimer. No patient with low/intermediate probability and negative D-dimer was diagnosed with pulmonary embolism (PE), regardless of the clinical scoring system used. Also of note is that a few patients with high probability scores did have $\mathrm{D}$-dimer testing (Table 2 ).

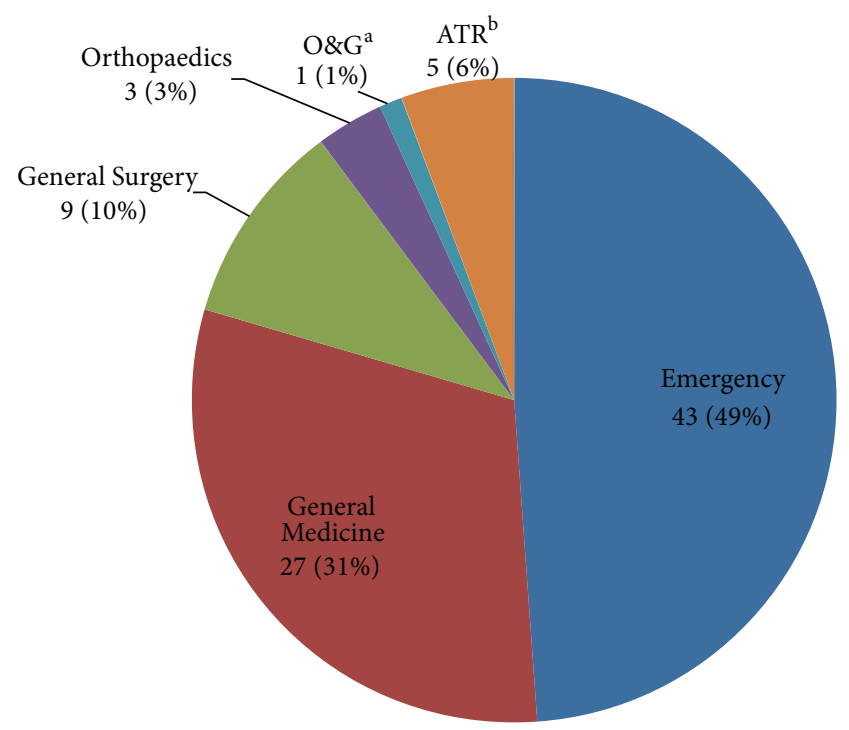

FIGURE 1: Referral source of the CTPA scans during the study period. This chart shows the number of CTPA scans requested from each department (total 88 scans). (a) Obstetrics and Gynaecology. (b) Assessment Treatment and Rehabilitation unit.

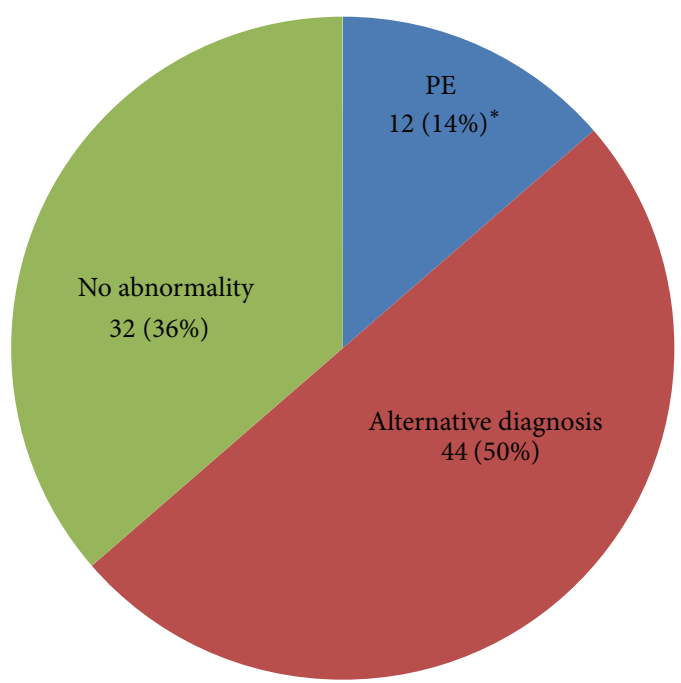

FIGURE 2: Findings of CTPA scans during the study period. ${ }^{*}$ Demonstrates the positive yield of CTPA in this audit.

\section{Discussion}

The positive yield of CTPA in Timaru Hospital is 14\%. The Royal College of Radiologist (UK) suggests $15.4-37.4 \%$ as an acceptable CTPA positive rate. The standard suggests an alternative diagnoses up to $56 \%$ [5]. However, positive rate of CTPA can vary according to the setting: it ranged from $7 \%$ to $13 \%$ in a few studies done in the Emergency Departments whereas an audit in Christchurch in 2006 revealed 30\% positive rate for patients admitted to the General Medicine, which may be explained by higher pretest probability in this patient group [6-10]. The positive rate also has decreased 
TABLE 1: Comparison of the number of CTPA requests, the number of inpatient admissions, and the frequency of CTPA by different referrers.

\begin{tabular}{lcccc}
\hline Referral source & No. of PE & No. of CTPA & Total no. of admissions* & No. of CTPA per 100 admissions \\
\hline Emergency & $\mathbf{6}$ & 43 & 7,093 & 0.61 \\
General Medicine & $\mathbf{3}$ & 27 & 1,496 & 1.80 \\
General Surgery & $\mathbf{1}$ & 9 & 1,338 & 0.67 \\
Orthopaedics & $\mathbf{1}$ & 3 & 405 & 0.74 \\
O\&G $^{\mathrm{a}}$ & $\mathbf{0}$ & 1 & 596 & 0.17 \\
ATR $^{\mathrm{b}}$ & $\mathbf{1}$ & 5 & 118 & 4.24 \\
\hline Total & $\mathbf{1 2}$ & 88 & 11,046 & 0.80 \\
\hline
\end{tabular}

${ }^{*}$ Represents the number of inpatient admissions in total for each department during the study period.

${ }^{a}$ Obstetrics and Gynaecology.

${ }^{\mathrm{b}}$ Assessment Treatment and Rehabilitation unit.

TABLE 2: Stratification of the CTPA requests according to the simplified Wells score and D-dimer $(n=88)$.

\begin{tabular}{lcccc}
\hline Simplified Wells score & \multicolumn{2}{l}{ D-dimer (Cut-off $=500 \mathrm{mcg} / \mathrm{L}$ FEU) } & $\begin{array}{c}\text { No. } \\
\text { of PE }\end{array}$ \\
\hline \multirow{3}{*}{ Low $(\leq 4)$} & 77 & Not done & $18^{*}$ & $1^{*}$ \\
& & Negative & $5^{\dagger}$ & $0^{\dagger}$ \\
& & Positive & 54 & 8 \\
\hline \multirow{3}{*}{ High $(>4)$} & \multirow{2}{*}{11} & Not done & 6 & 1 \\
& & Negative & 0 & 0 \\
& & Positive & 5 & 2 \\
\hline
\end{tabular}

${ }^{\S}$ Consists of previous pulmonary embolism or deep vein thrombosis, 1.5 point; heart rate $>100$ beats/minute, 1.5 point; recent surgery or immobilisation (within the last 30 days), 1.5 point; clinical signs of deep vein thrombosis, 3 points; alternative diagnosis less likely than pulmonary embolism, 3 points; haemoptysis, 1 point; and active cancer (treated within the last $6 \mathrm{mo}$ ), 1 point [2].

${ }^{*}$ Demonstrates that 18 scans were performed on patients with low Wells score who did not have D-dimer testing. One of these 18 scans showed PE.

${ }^{\dagger}$ Demonstrates that 5 scans were performed on patients with low Wells score with negative D-dimer. None of these 5 scans revealed PE.

over the years in general due to increasing use of CTPA as a screening tool without judicious patient selection [11].

The low yield is perhaps suggestive of overuse of CTPA. Or analyses showed that in contrast to published evidence, virtually no formal pretest probability scoring was performed. Also, despite the current evidence that argues against performing CTPA in patients with low/intermediate clinical probability and negative $\mathrm{D}$-dimer as mentioned above, $5(6 \%)$ scans were performed on such patients. Importantly none of these were diagnosed with $\mathrm{PE}$, validating the recommendation of the guidelines.

Our data further reveals that $18(20 \%)$ scans were performed on patients with low Wells scores without D-dimer testing, and one of these scans was positive for PE.

When the Revised Geneva scoring system was applied instead, 23 (26\%) scans were performed on patients with low/intermediate probability without $\mathrm{D}$-dimer testing, and two of these scans were positive for PE. Therefore, further $17-21$ (19-24\%) scans, depending on the probability scoring system used, could have been avoided assuming that $D$-dimer would have been negative if it had been tested. Adherence to the international guidelines therefore may have reduced CTPA requests at least by $5(6 \%)$ and potentially up to 26 $(30 \%)$ scans. By reducing the total number of scans from 88 to 62 , it is estimated that the positive yield of CTPA can improve from $14 \%$ to as high as $19 \%$. This would improve cost effectiveness and reduce the risk of contrast and radiation exposure.

D-dimer concentration has been shown to increase with age and can result in additional unnecessary CTPA referrals due to false positivity in elderly patients [12, 13]. Agedependent D-dimer cut-off has been shown to increase the number of cases in which PE can be safely excluded without need for CTPA [14, 15]. When our data was analysed using Douma and colleagues' age-dependent cut-off (calculated as age $\times 10 \mathrm{mcg} / \mathrm{L} \mathrm{FEU} \mathrm{for} 50$ years or older, otherwise $500 \mathrm{mcg} / \mathrm{L} \mathrm{FEU}$ ) [14], the number of negative $\mathrm{D}$-dimer in low/intermediate probability patients increased by onefrom five to six cases. In other words, the number of CTPA scans required decreased from 54 to 53 . We expect that the effect would be greater if the sample size was larger.

In our small district general hospital, $80 \%$ of the CTPA scans are requested from the Emergency and General Medicine Departments. It is interesting to note that only three (3\%) CTPA referrals were from orthopaedic department during the five-month period. One of the three referrals was positive for PE. This raised the questions whether CTPA is being underutilised for investigation of $\mathrm{PE}$ in orthopaedic patients. Although the positive yield does seem to be on high side in the orthopaedic department compared to the others, the sample size is too small to conclude. However, a prospective cohort study involving one million women revealed that women were 40 times more likely to develop venous thromboembolism during the first week after an inpatient operation compared to incident rates without surgery, peaking at 110 times during the third week after operation [16]. Hip/knee replacement surgery was associated with the greatest risk of VTE in the first six weeks among all the surgery types-220 times the risk without surgery [16].

It is also notable that ATR had the highest number of CTPA requests per admission, five times the local average. The figure is suggestive of overutilisation, although it may be due to the lower number of admissions due to lengthier hospital stays. It can also be explained by the significant number of orthopaedic and other post-op patients who were 
transferred to the ATR unit for rehabilitation. Although CTPA scans were not frequently requested while admitted to Orthopaedic Surgery, some patients were scanned when admitted to the rehabilitation facilities.

Limitations of the audit include small sample size, subjectivity of probability scoring, reviewer bias, and poor documentation in notes. Also, V/Q scan referrals have not been reviewed as it is not available in Timaru. However we believe that these observations are atypical for other nonacademic institutions.

In conclusion, the current trend in CTPA requests in Timaru Hospital for diagnosis of acute PE has room for improvement in particular in documenting a clinical probability score. Adherence to the current guidelines could have reduced CTPA scans required by $6-30 \%$, thereby improving the positive yield from $14 \%$. Extrapolated to a larger scale, this would translate into significantly reduction of cost, contrast contact, and radiation exposure.

\section{Acknowledgments}

The authors would like to acknowledge the support from the Medical Records for retrieving the patient files, the Coding Department for the data on the number of inpatient admissions, and the Radiology Department for encouragement and information on Timaru hospital's CT scanner.

\section{References}

[1] D. H. Newman and D. L. Schriger, "Rethinking testing for pulmonary embolism: less is more," Annals of Emergency Medicine, vol. 57, no. 6, pp. 622-627, 2011.

[2] B. T. Thompson and C. A. Hales, "Diagnosis of acute pulmonary embolism," UpToDate, 2012.

[3] I. A. Campbell, A. Fennerty, A. C. Miller et al., "BTS guidelines for the management of suspected acute pulmonary embolism," Thorax, vol. 58, no. 6, pp. 470-483, 2003.

[4] STA-Liatest, "D-Di: immuno-turbidimetric assay of D-dimer," Product Manual REF 00515, Diagnostica Stago SAS, 2011.

[5] MAking the Best Use of a CTPA, Guidelines for Doctors, Department of Clinical Radiology, The Royal College of Radiologist, 6th edition, 2007.

[6] J. A. Kline, D. M. Courtney, C. Kabrhel et al., "Prospective multicenter evaluation of the pulmonary embolism rule-out criteria," Journal of Thrombosis and Haemostasis, vol. 6, no. 5, pp. 772-780, 2008.

[7] F. S. Drescher, S. Chandrika, I. D. Weir et al., "Effectiveness and acceptability of a computerized decision support system using modified wells criteria for evaluation of suspected pulmonary embolism," Annals of Emergency Medicine, vol. 57, no. 6, pp. 613621, 2011.

[8] J. H. Donohoo, W. W. Mayo-Smith, J. A. Pezzullo, and T. K. Egglin, "Utilization patterns and diagnostic yield of 3421 consecutive multidetector row computed tomography pulmonary angiograms in a busy emergency department," Journal of Computer Assisted Tomography, vol. 32, no. 3, pp. 421-425, 2008.

[9] E. G. Stein, L. B. Haramati, M. Chamarthy, S. Sprayregen, M. M. Davitt, and L. M. Freeman, "Success of a safe and simple algorithm to reduce use of CT pulmonary angiography in the emergency department," American Journal of Roentgenology, vol. 194, no. 2, pp. 392-397, 2010.

[10] P. Chin, M. Hurrell, D. McGregor, and L. Beckert, "The role of CT pulmonary angiography in patients with suspected pulmonary embolism admitted to general medicine," New Zealand Medical Journal, vol. 119, no. 1237, 2006.

[11] J. Tan, A. Pais, and T. Jain, “The appropriate use of computed tomography pulmonary angiogram in diagnosing pulmonary embolism," OJCA Online, vol. 4, no. 1, 2012.

[12] P. L. Harper, E. Theakston, J. Ahmed, and P. Ockelford, "Ddimer concentration increases with age reducing the clinical value of the $\mathrm{D}$-dimer assay in the elderly," Internal Medicine Journal, vol. 37, no. 9, pp. 607-613, 2007.

[13] M. Righini, G. Le Gal, A. Perrier, and H. Bounameaux, "The challenge of diagnosing pulmonary embolism in elderly patients: influence of age on commonly used diagnostic tests and strategies," Journal of the American Geriatrics Society, vol. 53, no. 6, pp. 1039-1045, 2005.

[14] R. A. Douma, G. le Gal, M. Söhne et al., "Potential of an age adjusted D-dimer cut-off value to improve the exclusion of pulmonary embolism in older patients: a retrospective analysis of three large cohorts," BMJ, vol. 340, article c1475, 2010.

[15] H. J. Schouten, H. L. Koek, R. Oudega et al., "Validation of two age dependent $\mathrm{D}$-dimer cut-off values for exclusion of deep vein thrombosis in suspected elderly patients in primary care: retrospective, cross sectional, diagnostic analysis," BMJ, vol. 344, article e2985, 2012.

[16] S. Sweetland, J. Green, B. Liu et al., "Duration and magnitude of the postoperative risk of venous thromboembolism in middle aged women: prospective cohort study," BMJ, vol. 339, article b4583, 2009. 


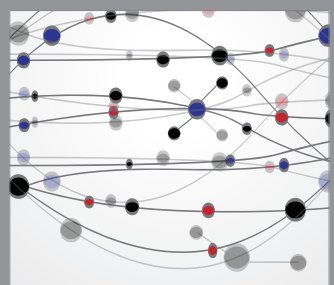

The Scientific World Journal
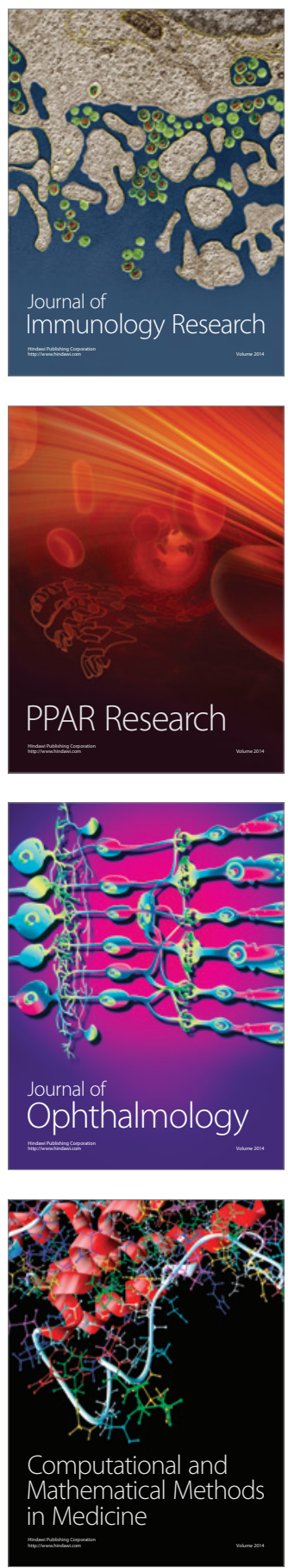

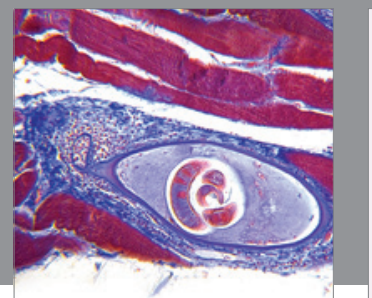

Gastroenterology

Research and Practice
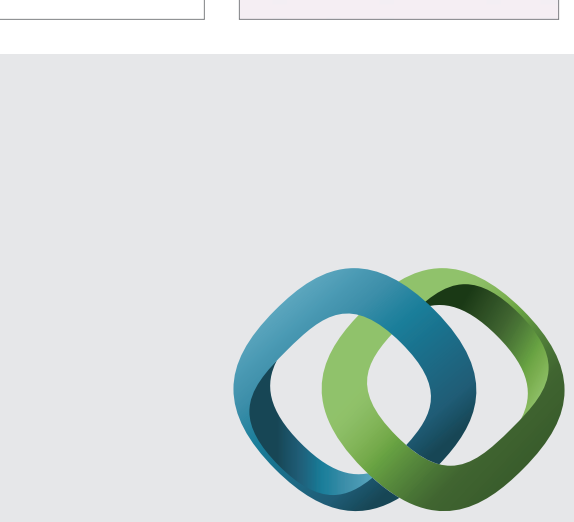

\section{Hindawi}

Submit your manuscripts at

http://www.hindawi.com
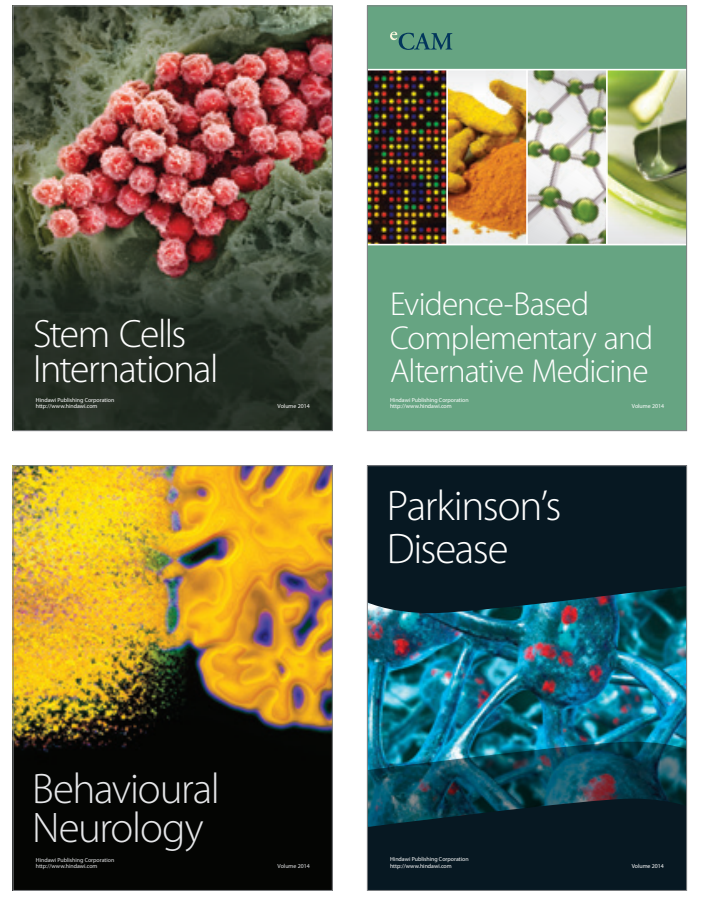
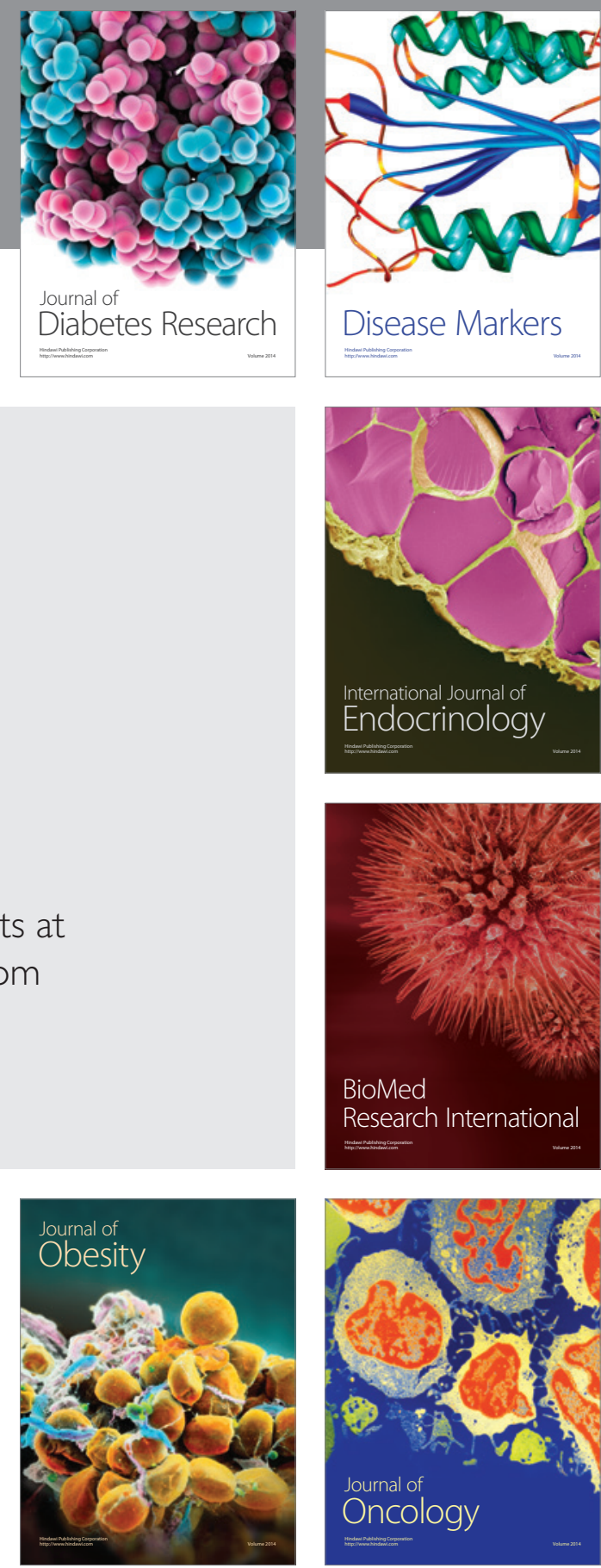

Disease Markers
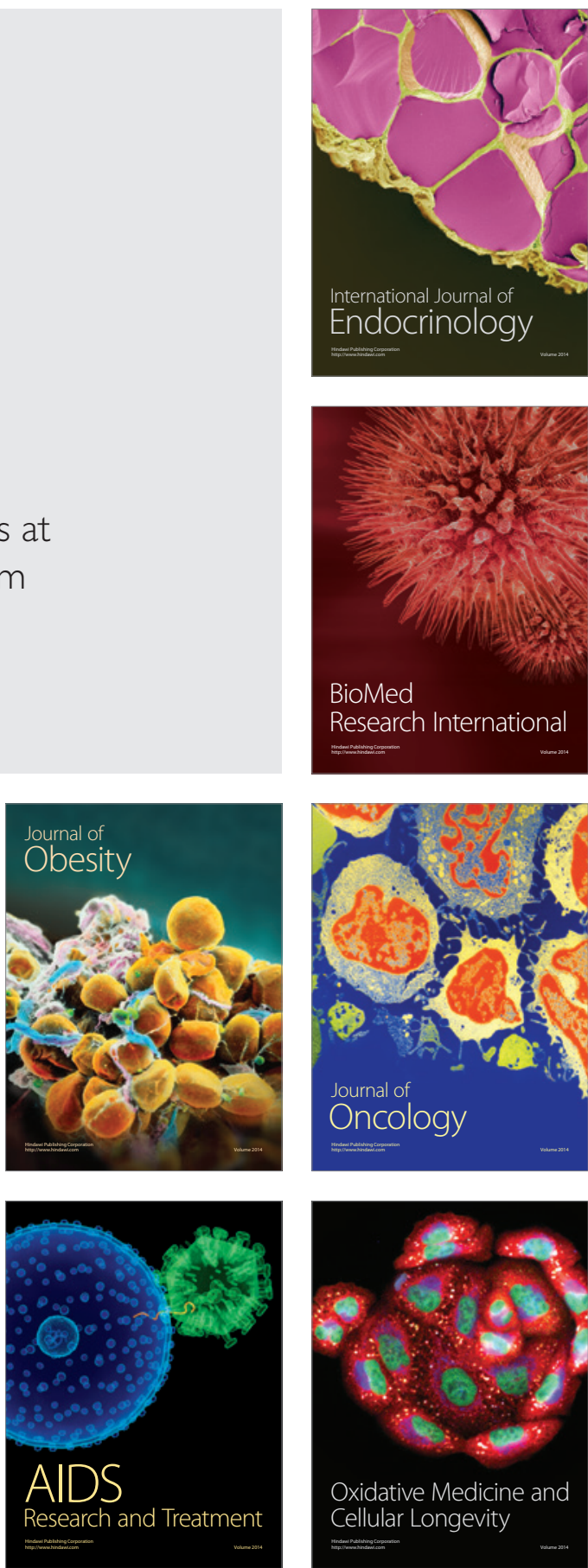